\title{
Renal function in patients with obstructive sleep apnea
}

\author{
Tomoyuki Kawada ${ }^{1}$
}

Received: 26 October 2016 / Accepted: 26 January 2017 / Published online: 3 February 2017

(C) Springer-Verlag Berlin Heidelberg 2017

Dear Editor,

Uyar et al. investigated the association between estimated glomerular filtration rate (eGFR) and obstructive sleep apnea (OSA) in 634 OSA patients aged 18 years or older [1]. eGFR was calculated with the Chronic Kidney Disease Epidemiology Collaboration (CKD-EPI) equation. Sixty-two subjects without OSA (controls) were also set for the analysis. The authors observed that eGFR values were significantly lower in OSA patients with metabolic syndrome (MetS), hypertension, and/or left ventricular hypertrophy. I have some concerns on their study.

First, the authors did not conduct multivariate analysis with adjustment of confounders. As MetS was associated with renal dysfunction, they speculated that renal dysfunction was derived from OSA and/or MetS. I think that comprehensive analyses with enough number of subjects would make stable risk assessment possible. On this point, Ogna et al. evaluated the association between early stages of CKD and sleep disordered breathing (SDB), restless legs syndrome (RLS), and subjective and objective sleep quality (SQ) in 1760 adults with sleep polysomnography at home [2]. They adopted multivariate logistic or linear regression analyses. Patients with early stages of CKD have impaired SQ, use more hypnotic drugs, and have an increased prevalence of SDB and PLM, but significant association with declining kidney function was only observed in objective SQ and PLMI by controlling for confounders. Namely, association between SDB/OSA and renal function was weak and not always observed. Although

Tomoyuki Kawada

kawada@nms.ac.jp

1 Department of Hygiene and Public Health, Nippon Medical School, 1-1-5 Sendagi, Bunkyo-Ku, Tokyo 113-8602, Japan biological mechanism on the association would be complicated, a follow-up study or an interventional study should be conducted to confirm causal association.

Relating to the first query, Chen et al. conducted a metaanalysis on the effect of positive airway pressure (PAP) on GFR in patients with SDB [3]. Although there was no change of GFR before and after PAP treatment in SDB patients, subgroup analyses presented a significant improvement of GFR in patients aged 55 years or older and patients with therapeutic duration of 3 months or longer.

Second, mean value of eGFR in their study did not decrease significantly according to the severity of OSA. In addition, mean value eGFR in patients with OSA was $90.74 \mathrm{ml} /$ $\min / 1.73 \mathrm{~m}^{2}$ in males and $87.73 \mathrm{ml} / \mathrm{min} / 1.73 \mathrm{~m}^{2}$ in females. I suppose that the authors handled patients without severe chronic renal disease. The association should also be evaluated by considering the severity of CKD in further study.

\section{Compliance with ethical standards}

Disclosure statement The author has indicated no financial support.

Conflict of interest The author declares that he has no conflict of interest.

\section{References}

1. Uyar M, Davutoğlu V, Gündoğdu N, Kosovalı D, Sarı İ (2016) Renal functions in obstructive sleep apnea patients. Sleep Breath 20(1):191-195

2. Ogna A, Forni Ogna V, Haba Rubio J et al (2016) Sleep characteristics in early stages of chronic kidney disease in the HypnoLaus cohort. Sleep 39(4):945-953

3. Chen LD, Lin L, Ou YW et al. (2016) Effect of positive airway pressure on glomerular filtration rate in patients with sleepdisordered breathing: a meta-analysis. Sleep Breath 2016 doi:10. 1007/s11325-016-1364-6 